\title{
Exercise during and after neoadjuvant rectal cancer treatment (the EXERT trial): study protocol for a randomized controlled trial
}

Andria R. Morielli ${ }^{1}$, Nawaid Usmani², Normand G. Boulé ${ }^{1}$, Diane Severin², Keith Tankel², Tirath Nijjar², Kurian Joseph ${ }^{2}$, Alysa Fairchild ${ }^{2}$ and Kerry S. Courneya ${ }^{1 *}$

\begin{abstract}
Background: Standard treatment for locally advanced rectal cancer includes 5-6 weeks of neoadjuvant chemoradiotherapy (NACRT) followed by total mesorectal excision 6-8 weeks later. NACRT improves local disease control and surgical outcomes but also causes side effects including fatigue, diarrhea, hand-foot syndrome, and physical deconditioning that may impede quality of life (QoL), treatment completion, treatment response, and long-term prognosis. Interventions to improve treatment outcomes and manage side effects that are safe, tolerable and low-cost are highly desirable. Exercise has been shown to improve some of these outcomes in other cancer patient groups but no study to date has examined the potential benefits (and harms) of exercise training during and after NACRT for rectal cancer.

Methods/design: The Exercise During and After Neoadjuvant Rectal Cancer Treatment (EXERT) trial is a single-center, prospective, two-armed, phase II randomized controlled trial designed to test the preliminary efficacy of exercise training in this clinical setting and to further evaluate its feasibility and safety. Participants will be 60 rectal cancer patients scheduled to receive long-course NACRT followed by total mesorectal excision. Participants will be randomly assigned to exercise training or usual care. Participants in the exercise training group will be asked to complete three supervised, high-intensity interval training sessions/week during NACRT and $\geq 150 \mathrm{~min} /$ week of unsupervised, moderate-to-vigorous-intensity, continuous exercise training after NACRT prior to surgery. Participants in the usual care group will be asked not to increase their exercise from baseline. Assessments will be completed pre NACRT, post NACRT, and pre surgery. The primary endpoint will be cardiorespiratory fitness $\left(\mathrm{VO}_{2}\right.$ peak) at the post-NACRT time point assessed by a graded exercise test. Secondary endpoints will include functional fitness assessed by the Senior's Fitness Test, QoL assessed by the European Organisation of Research and Treatment of Cancer, and symptom management assessed by the M.D. Anderson Symptom Inventory. Exploratory clinical endpoints will include treatment toxicities, treatment completion, treatment response, and surgical complications.
\end{abstract}

Discussion: If the preliminary findings of EXERT are positive, additional research will be warranted to confirm whether exercise is an innovative treatment to maintain QoL, manage side effects, and/or improve treatment outcomes in rectal cancer patients.

Trial registration: ClinicalTrials.gov, ID: NCT03082495. Registered on 9 February, 2017.

Keywords: Exercise, Cancer, Symptom management, Quality of life, Physical fitness, Physical activity

\footnotetext{
* Correspondence: Kerry.courneya@ualberta.ca

${ }^{1}$ Faculty of Kinesiology, Sport, and Recreation, University of Alberta, 1-113

University Hall, Van Vliet Complex, Edmonton, AB T6G 2H9, Canada

Full list of author information is available at the end of the article
} 


\section{Background}

Current standard treatment for locally advanced rectal cancer (stages II and III) includes long-course (5-6 weeks) neoadjuvant chemoradiotherapy (NACRT) followed by surgical resection using total mesorectal excision 6-8 weeks later [1]. When compared to postoperative chemoradiotherapy, NACRT improves local recurrences rates and may improve surgical outcomes in some patients [2, 3]. Unfortunately, NACRT causes acute toxicities including fatigue, diarrhea, hand-foot syndrome, hematologic toxicity, cardiotoxicity, and physical deconditioning that can cause declines in quality of life (QoL) [4] and may even impede treatment completion, treatment response, and long-term prognosis. Safe, tolerable, and low-cost interventions to manage these side effects and improve treatment outcomes in this clinical setting are highly desirable. We propose that an exercise training intervention initiated during NACRT in patients with rectal cancer may improve cardiorespiratory fitness, symptom management, QoL, treatment completion, treatment response, surgical complications, and possibly even survival (Fig. 1).

Exercise has been shown to manage some side effects and improve QoL in several cancer patient groups receiving adjuvant therapy [5]. Moreover, limited research has suggested that exercise during chemotherapy for some cancer patient groups may improve chemotherapy completion rates [6-8], treatment response [9], and even long-term survival [10]. Additionally, there is some evidence that pre-operative exercise may improve fitness and surgical outcomes in cancer patients [11]. Finally, preliminary evidence has suggested that exercise is feasible and safe in the neoadjuvant setting [12].

Despite the emerging evidence for the benefits of exercise in some cancer patient groups receiving some treatment protocols, only preliminary research has examined exercise in rectal cancer patients during and after
NACRT. Similar to drug trials, exercise trials have demonstrated that research in one cancer patient/treatment group rarely generalizes to another cancer patient/treatment group [13]. Preliminary research suggests that exercise initiated immediately after NACRT is feasible and may improve cardiorespiratory fitness [14], which has prompted a phase II trial in this clinical space [15]. Furthermore, two phase I studies [16, 17], including one from our group [16], have demonstrated the preliminary feasibility and safety of exercise during NACRT for rectal cancer patients. Finally, one ongoing randomized controlled trial is examining the feasibility of an unsupervised walking program both during and after NACRT (ISRCTN62859294). Here, we propose the Exercise During and After Neoadjuvant Rectal Cancer Treatment (EXERT) trial which, to our knowledge, is the first phase II trial designed to examine the preliminary efficacy of exercise training in rectal cancer patients during and after NACRT.

\section{Study objectives \\ Primary objective}

The primary objective of the EXERT trial is to examine the effects of a supervised, high-intensity interval training (HIIT) program, compared to usual care, on cardiorespiratory fitness during NACRT.

\section{Secondary objectives}

The secondary objectives of the EXERT trial are to (1) compare an unsupervised, moderate-to-vigorous-intensity, continuous exercise training program after NACRT to usual care on cardiorespiratory fitness, (2) compare the supervised HIIT program during NACRT and unsupervised continuous exercise training program after NACRT on functional fitness, QoL, and symptom burden, (3) establish the feasibility and safety of the supervised HIIT program during NACRT, and (4) investigate

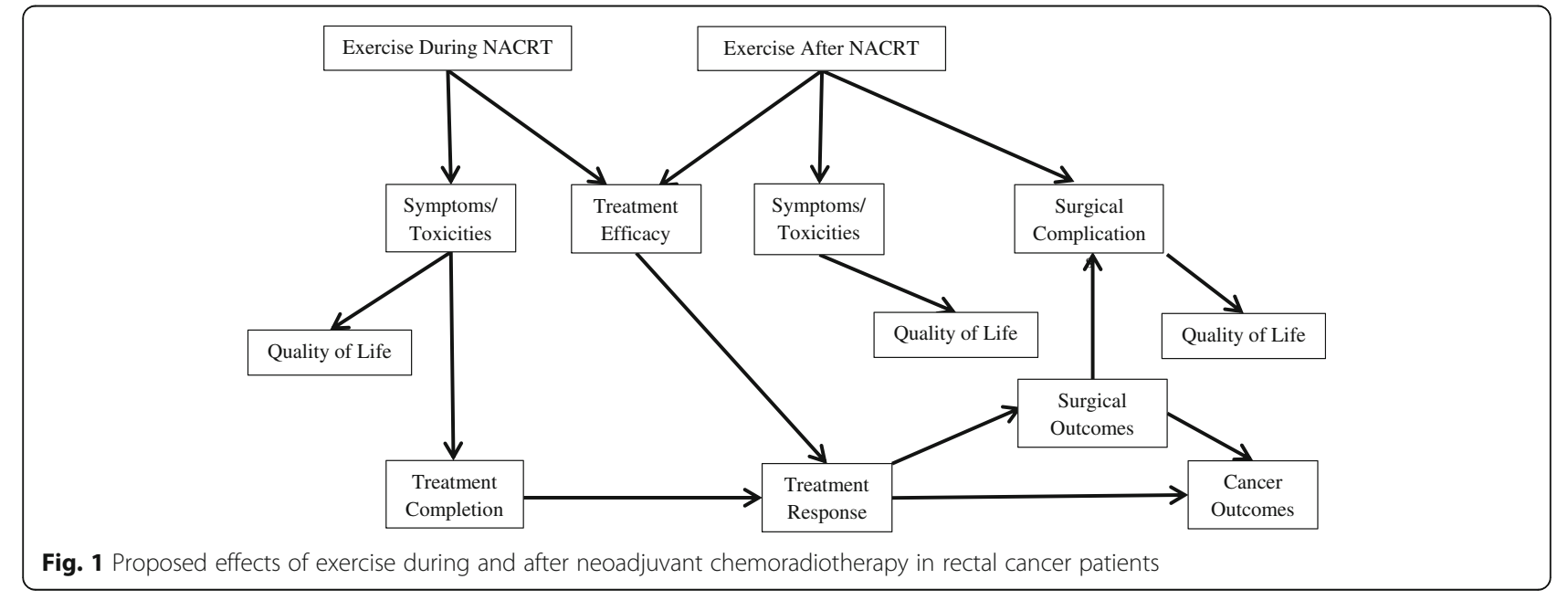


the motivational outcomes and determinants of exercise during and after NACRT.

\section{Exploratory objectives}

The exploratory objectives of the EXERT trial are to compare a supervised HIIT program during NACRT followed by an unsupervised, moderate-to-vigorous-intensity, continuous exercise training program after NACRT to usual care on clinical outcomes including treatment toxicities, treatment completion, treatment response, and surgical complications.

\section{Methods/design}

\section{Study design}

The EXERT trial will be a single-center, prospective, twoarmed, phase II randomized controlled trial conducted in Edmonton, Alberta. The EXERT trial has been approved by the Health Research Ethics Board of Alberta-Cancer
Committee and all participants will be required to provide written informed consent. The proposed participant flow through the study is shown in Fig. 2. Health-related fitness outcomes and patient-reported outcomes will be assessed at baseline (0-7 days before starting NACRT), post NACRT (0-7 days after completing NACRT), and pre surgery (7-14 days before the planned surgery date). The Standard Protocol Items: Recommendations for Interventional Trials (SPIRIT) Figure for the EXERT trial is shown in Fig. 3 (Additional file 1).

\section{Eligibility criteria}

Men and women will be eligible for the trial if they (1) are $\geq 18$ years old, (2) are scheduled to received standard NACRT consisting of 5-6 weeks of radiotherapy (45-54 Gy) with concurrent chemotherapy (orally administered capecitabine or intravenously administered 5-fluorarcil) followed by total mesorectal excision, (3)

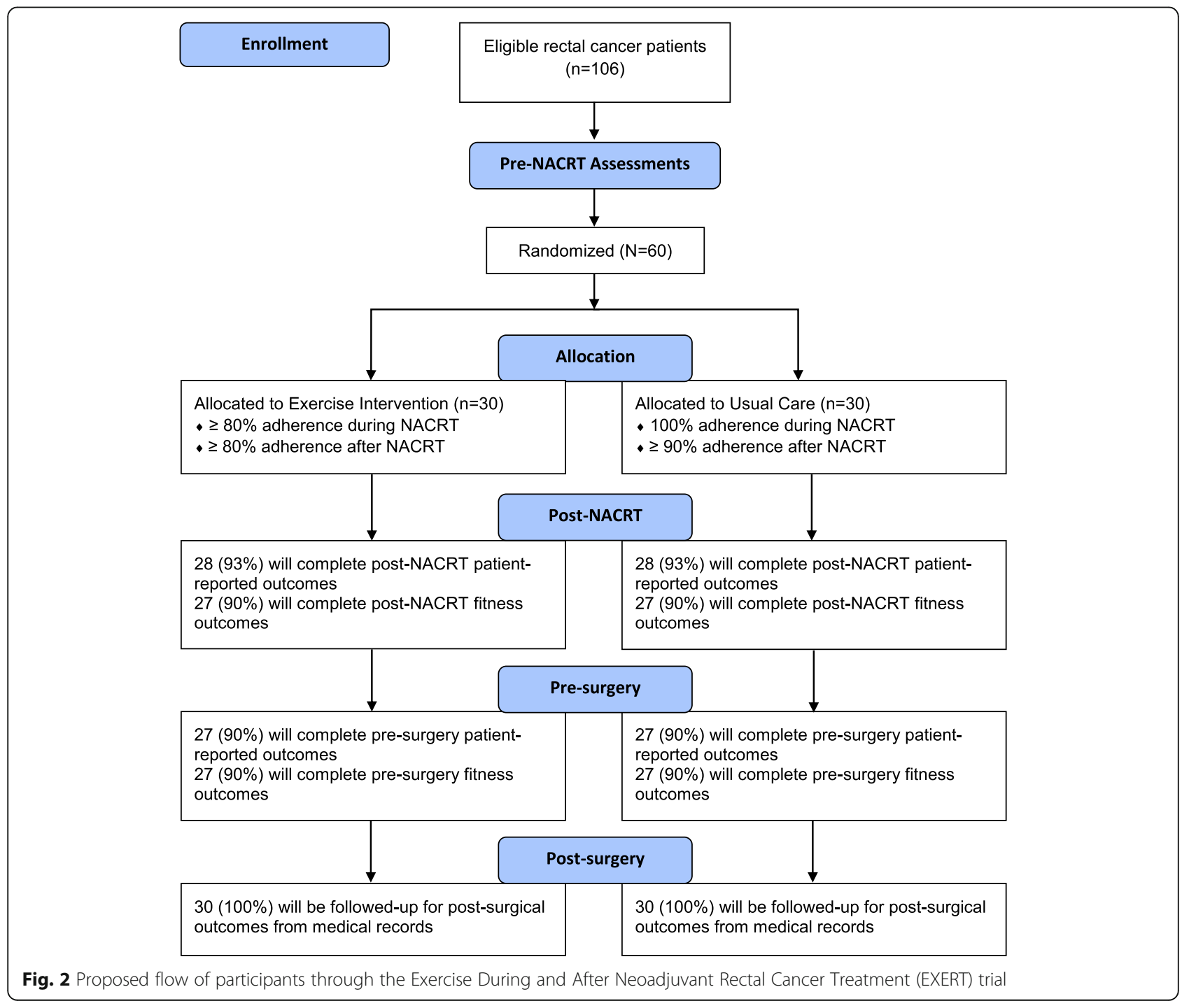




\begin{tabular}{|c|c|c|c|c|c|c|c|}
\hline \multirow[b]{3}{*}{ TIMEPOINT } & \multicolumn{6}{|c|}{ STUDY PERIOD } & \multirow[b]{3}{*}{$t_{5}$} \\
\hline & $\begin{array}{l}\text { Enrolment \& } \\
\text { Baseline }\end{array}$ & Allocation & \multicolumn{3}{|c|}{ Post-allocation } & \multirow{2}{*}{$\begin{array}{c}\text { Close-out } \\
t_{4}\end{array}$} & \\
\hline & $-t_{1}$ & 0 & $t_{1}$ & $t_{2}$ & $t_{3}$ & & \\
\hline & Pre-NACRT & Pre-NACRT & $\begin{array}{c}\text { Exercise } \\
\text { during } \\
\text { NACRT } \\
\end{array}$ & $\begin{array}{c}\text { Post- } \\
\text { NACRT } \\
\text { Assessment } \\
\end{array}$ & $\begin{array}{c}\text { Exercise } \\
\text { post- } \\
\text { NACRT } \\
\end{array}$ & $\begin{array}{l}\text { Pre-surgery } \\
\text { Assessment }\end{array}$ & $\begin{array}{c}\begin{array}{c}\text { Post- } \\
\text { surgery } \\
\text { follow-up }\end{array} \\
\end{array}$ \\
\hline \multicolumn{8}{|l|}{ ENROLMENT: } \\
\hline \multirow{3}{*}{$\begin{array}{l}\text { Eligibility screen } \\
\text { Informed consent } \\
\text { Randomization }\end{array}$} & $\mathrm{X}$ & & & & & & \\
\hline & $x$ & & & & & & \\
\hline & & $x$ & & & & & \\
\hline \multicolumn{8}{|l|}{ INTERVENTIONS: } \\
\hline \multicolumn{8}{|l|}{ Exercise Training } \\
\hline \multicolumn{8}{|l|}{ Usual Care } \\
\hline \multirow{3}{*}{$\begin{array}{r}\text { ASSESSMENTS: } \\
\text { Primary outcome: } \\
\text { Cardiorespiratory } \\
\text { fitness. } \\
\text { Secondary } \\
\text { outcomes: } \\
\text { Functional fitness, } \\
\text { quality of life, } \\
\text { symptom burden, } \\
\text { and exercise } \\
\text { motivation. }\end{array}$} & & & & & & & \\
\hline & $\mathrm{x}$ & & & $\mathrm{X}$ & & $\mathrm{X}$ & \\
\hline & $x$ & & & $x$ & & $x$ & \\
\hline \multirow{2}{*}{$\begin{array}{r}\text { Feasibility and } \\
\text { safety outcomes } \\
\text { Exploratory } \\
\text { outcomes: } \\
\text { Treatment toxicities, } \\
\text { treatment } \\
\text { completion, } \\
\text { treatment response, } \\
\text { and surgical } \\
\text { outcomes and } \\
\text { complications. }\end{array}$} & $\mathrm{X}$ & $\mathrm{x}$ & $x$ & $x$ & $x$ & $x$ & $x$ \\
\hline & & & $\mathrm{x}$ & $\mathrm{x}$ & $\mathrm{x}$ & $\mathrm{x}$ & $\mathrm{x}$ \\
\hline
\end{tabular}

Fig. 3 Standard Protocol Items: Recommendations for Interventional Trials (SPIRIT) Figure for the Exercise During and After Neoadjuvant Rectal Cancer Treatment (EXERT) trial

receive medical clearance to participate in the study as determined by their treating oncologist, the Physical Activity Readiness Questionnaire for Everyone (PAR-Q+), and a certified exercise physiologist, (4) are able to complete the pre-NACRT graded exercise test, (5) are not currently engaging in any regular vigorous-intensity exercise and/ or $\geq 150 \mathrm{~min}$ of moderate-intensity-exercise/week, (6) are able to provided written informed consent and complete questionnaires in English, and (7) are willing to be randomized to exercise training or usual care (no exercise) for 12 weeks.

\section{Recruitment}

Prospective patients will be approached by the treating radiation oncologist and study coordinator at the time of their initial radiation consultation. The study coordinator will follow-up with eligible patients by phone and schedule interested patients for pre-NACRT testing. This recruitment strategy was effective in our feasibility study with 18 of 32 patients (56\%) being recruited over a 6-month period [16].

\section{Randomization and blinding}

After completing all baseline assessments, patients will be randomly assigned to either the exercise training group or usual care group in a 1:1 ratio using block randomization. A research assistant, not otherwise involved in the trial, will generate the block sizes and randomization sequence using a computer-generated random allocation sequence which will be concealed from the recruiting study coordinator. Given the nature of the intervention, it is not possible to blind the investigators or participants to group allocation. Additionally, due to logistical challenges at our facility, it is difficult to 
blind outcome assessors to group allocation for the primary outcome of cardiorespiratory fitness and the secondary outcomes of functional fitness. Nevertheless, fitness outcome assessors will follow a detailed protocol and be trained in the importance of standardizing outcome assessments and avoiding bias. Moreover, outcome assessors will not review the metabolic data during the cardiorespiratory fitness test, and the criteria for achieving peak volume of oxygen consumption $\left(\mathrm{VO}_{2}\right.$ peak) will undergo an independent review. Finally, outcome assessors will be blinded to group assignment for the exploratory outcomes of treatment toxicities, treatment completion, treatment response, and surgical complications which will be assessed by medical staff not otherwise involved in the study.

\section{Intervention}

For patients randomized to the exercise training group, the intervention will be divided into two phases: (1) during NACRT and (2) post NACRT. During NACRT, all of the exercise sessions will be supervised by a certified exercise physiologist. We previously determined that it was feasible for patients to attend supervised exercise sessions at our fitness center (within a 5-min walk from the cancer center) since they were already coming to the cancer center 5 days/week for radiation treatment [16]. During NACRT, patients will be asked to complete 18 supervised HIIT sessions (i.e., three sessions/week for 6 weeks) and to continue with any light-to-moderate-intensity exercise that they were performing at baseline. We selected a HIIT program for evaluation because of its ability to maximize cardiorespiratory fitness improvements over a short period of time [18]. Moreover, HIIT has previously demonstrated safety and feasibility in clinical populations including patients with cardiometabolic disease [19], diabetes [20], and during adjuvant chemotherapy in patients with mixed cancers [21]. HIIT is characterized by relatively short bursts of vigorousintensity exercise, interspersed by periods of rest or light-intensity exercise for recovery. There are an endless number of possible combinations that can make up a HIIT program; however, HIIT typically refers to exercise intensities corresponding to $\geq 85 \%$ of peak heart rate or $\geq 80 \%$ of $\mathrm{VO}_{2}$ peak [19]. We have designed the HIIT program in the EXERT trial to closely match a previously published HIIT program which has demonstrated feasibility, safety, and greater improvements in cardiorespiratory fitness in patients with coronary artery disease [22].

In our phase I study, we demonstrated an excellent median attendance rate of $83 \%$ to three sessions/week of moderate-intensity, continuous exercise training during NACRT [16]. Moreover, no adverse events were observed and our evaluation was that even higher-intensity exercise training would be feasible in this clinical setting. Nevertheless, the safety and feasibly of HIIT during NACRT in rectal cancer patients has yet to be established and is a key objective of our study.

In our feasibility study, the most frequently used modality was the treadmill (67.4\% of sessions) [16]. Thus, the HIIT program will consist of uphill treadmill walking. Each HIIT session will start with a 5-min warm-up at a workload that elicits $30-40 \%$ of $\mathrm{VO}_{2}$ peak during the baseline graded exercise test. Patients will complete 2min, high-intensity intervals at a workload that elicits $85 \%$ of $\mathrm{VO}_{2}$ peak during the baseline graded exercise test. Between the high-intensity intervals, the activerecovery intervals will consist of $2 \mathrm{~min}$ at a workload that elicits $40 \%$ of $\mathrm{VO}_{2}$ peak during the baseline graded exercise test. Each HIIT exercise session will end with a 5 -min cool-down totaling $40 \mathrm{~min} / \mathrm{session}$. The number of HIIT intervals will begin at five and progress by one every second session up to eight intervals (Table 1).

Prior to each exercise session, an exercise specialist will assess blood pressure and heart rate and ask patients to report any immediate symptoms. Additionally, body temperature will be assessed in patients reporting any signs or symptoms of a fever. If body temperature is $\geq 38{ }^{\circ} \mathrm{C}$, patients will be instructed not to exercise that day. For each supervised session, the exercise specialist will record attendance and the workload (i.e., treadmill speed and incline), Rating of Perceived Exertion (RPE) (Borg 0-10), and heart rate for each

Table 1 High-intensity interval training program during neoadjuvant chemoradiotherapy in the Exercise During and After Neoadjuvant Rectal Cancer Treatment (EXERT) trial

\begin{tabular}{|c|c|c|c|c|c|c|c|}
\hline \multirow{3}{*}{ Session } & \multicolumn{3}{|c|}{ Interval period } & \multicolumn{3}{|c|}{ Recovery period } & \multirow{3}{*}{$\begin{array}{l}\text { Total duration } \\
\text { (min) }\end{array}$} \\
\hline & No. & Duration & Intensity & No. & Duration & Intensity & \\
\hline & & $(\min )$ & $\% \mathrm{VO}_{2}$ peak $^{\mathrm{a}}$ & & $(\min )$ & $\% \mathrm{VO}_{2}$ peak $^{\mathrm{a}}$ & \\
\hline $1-2$ & 5 & 2 & 85 & 4 & 2 & 40 & 28 \\
\hline $3-4$ & 6 & 2 & 85 & 5 & 2 & 40 & 32 \\
\hline $5-6$ & 7 & 2 & 85 & 6 & 2 & 40 & 36 \\
\hline $7-18$ & 8 & 2 & 85 & 7 & 2 & 40 & 40 \\
\hline
\end{tabular}

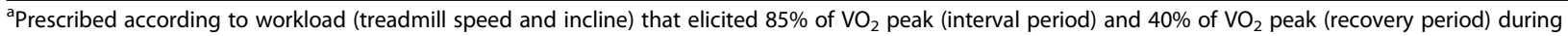
baseline graded exercise test

Note: All HIIT sessions start with a 5-min warm-up and end with a 5-min cool-down 
high-intensity interval. Optimal adherence to the supervised exercise sessions will be facilitated by scheduled appointments, flexibility in scheduling the exercise sessions (i.e., according to patients' radiation sessions), immediate follow-up and re-booking of missed sessions, personable exercise trainers, and free parking. In our feasibility study, we identified the most common barriers to exercising during NACRT as side effects from chemoradiotherapy $(88 \%)$, fatigue $(76 \%)$, and diarrhea (71\%) [23]. In the EXERT trial, we will optimize adherence to the supervised exercise sessions by modifying each session according to any immediate symptoms or side effects that patients are experiencing.

If a patient is experiencing immediate side effects that hinder their ability to complete the high-intensity intervals at the prescribed workload, the fitness attendant will modify the exercise session according to what the patient is able and willing to do. Options for modifying the exercise dose will include either reducing the workload of the high-intensity intervals, reducing the number of high-intensity intervals, or both. All reasons for dose modification will be noted.

After NACRT, patients will be asked to complete at least $150 \mathrm{~min}$ of unsupervised, moderate-to-vigorousintensity, continuous exercise training training/week (current Canadian Physical Activity Guidelines). In our phase I study, it was feasible for patients to achieve 150 min of mostly unsupervised, moderate-intensity exercise/week after NACRT. Although local patients in our pilot study were offered a supervised exercise program after NACRT, only two out of 16 patients expressed interest in continuing with supervised exercise in this phase and patients mainly achieved their weekly exercise minutes by walking outdoors or by using pre-existing home exercise equipment (e.g., treadmill, elliptical, and upright bike). For this reason, we felt that it would be difficult to deliver a standardized and replicable HIIT program after NACRT. Moreover, in our phase I study, cardiorespiratory fitness improved by $2.4 \mathrm{ml} / \mathrm{kg} / \mathrm{min}$ from post NACRT to pre surgery suggesting that an unsupervised, moderateintensity, continuous exercise training program may be effective at improving cardiorespiratory fitness after NACRT and prior to surgery in rectal cancer patients. Finally, after our experience in the phase I study, we felt that it would be feasible and safe for rectal cancer patients to complete moderate-to-vigorous-intensity continuous exercise in this phase and may result in greater improvements in cardiorespiratory fitness. Nevertheless, one of the goals of the EXERT trial is to further establish the feasibility and safety of continuous exercise training after NACRT and to determine its preliminary efficacy at improving outcomes for rectal cancer patients.
After NACRT, the exercise will be individualized according to patients' post-NACRT graded exercise test (i.e., heart rate that corresponded with approximately 46-91\% of $\mathrm{VO}_{2}$ peak) [24]. Patients will be provided with a heart rate monitor and will also be instructed on how to use RPE and the talk-test to determine the intensity of their exercise sessions. Patients will be provided with examples of how to complete the exercise (e.g., $30 \mathrm{~min}, 5$ days/week; $50 \mathrm{~min}, 3$ days/week). Moreover, patients will be instructed that the exercise completed in this phase should be in addition to what they were already doing at baseline. Finally, patients will receive printout materials with instructions on how to complete the exercise in the post-NACRT phase as well an exercise log to help them keep track of their exercise. After NACRT, optimal adherence will be achieved using a more formal behavioral support program based on the Theory of Planned Behavior (TPB) [25]. The study coordinator will maintain weekly contact with each patient via telephone and offer behavioral support sessions consisting of standard behavioral change techniques including goal setting, planning, self-monitoring, and overcoming barriers.

\section{Usual care group}

Patients randomized to the usual care group will receive standard medical care which includes meeting with a dietician weekly to ensure adequate caloric and nutrient intake. Patients in the usual care group will be asked not to increase their physical activity/exercise levels during or after NACRT. Exercise is not currently part of standard care for these patients at our center and patients do not receive any exercise recommendations. After the pre-surgery assessment, patients in the usual care group will be offered a copy of the Canadian Physical Activity Guidelines and encouraged to initiate an exercise program after they recover from surgery and receive medical clearance from their physician.

\section{Outcome measures \\ Primary outcome measure}

We selected cardiorespiratory fitness as the primary endpoint for the EXERT trial because there is clinical equipoise as to whether 6 weeks of HIIT during NACRT is sufficient to meaningfully improve cardiorespiratory fitness. Moreover, cardiorespiratory fitness is an established surrogate for some patient-reported outcomes and clinical outcomes [9, 26-29]. Our primary measure of cardiorespiratory fitness, $\mathrm{VO}_{2}$ peak, will be assessed by the modified Bruce graded exercise test on a treadmill with direct measures of cardiorespiratory variables using a metabolic measurement system (Parvo Medics TrueOne 2400; Sandy, UT, USA ) [30, 31]. The modified Bruce treadmill protocol was designed for use in 
high-risk and elderly individuals. Briefly, the protocol will start at $1.7 \mathrm{mph}$ and $0 \%$ grade and will progress every 3 min until the patient reaches volitional fatigue or if any exercise contraindications occur. During the test, heart rate will be monitored continuously and recorded every minute and blood pressure, oxygen saturation, and rating of perceived exertion will be measured and recorded in the last minute of every stage. Immediately after the test, patients will complete a 5-min active recovery $(1.7 \mathrm{mph}$ and $0 \%$ grade). During the active recovery, heart rate, blood pressure, and oxygen saturation will continue to be monitored and recorded at $1 \mathrm{~min}$ and $5 \mathrm{~min}$.

\section{Secondary outcome measures}

Functional fitness will be assessed by the Senior's Fitness Test which measures basic mobility-related parameters associated with functional abilities in the everyday living of older adults [32, 33]. The Senior's Fitness Test consists of six items including the 30-s chair stand (assessment of lower body strength), the arm curl (assessment of upper body strength), the chair sit-and-reach (assessment of lower body flexibility), the back scratch (assessment of upper body flexibility), the 8-foot up-and-go (assessment of agility and dynamic balance), and the 6min walk (assessment of aerobic endurance).

Quality of life will be assessed by the widely used and validated European Organisation of Research and Treatment of Cancer (EORTC) core 30-item questionnaire (QLQ-C30) version 3.0 [34]. We selected the EORTC QLQ-C30 because it assesses symptoms, physical function, psychosocial function, and overall QoL [35]. The EORTC QLQ-C30 is composed of five multi-item functional scales (physical, cognitive, role, emotional, and social), three multi-item symptom scales (fatigue, nausea and vomiting, and pain), five single-item symptom scales (dyspnea, insomnia, loss of appetite, constipation, and diarrhea), a single-item financial impact scale, and a two-item global health and QoL scale. Additionally, the EORTC-QLQ-CR29 (colorectal cancer) will be used to assess QoL. The EORTC QLQ-CR29 has demonstrated acceptable validity and reliability for its supplemental use with the QLQ-C30 to assess the QoL of colorectal cancer patients during treatment [36]. The EORTC QLQ-CR29 contains 29 questions and evaluates urinary dysfunction, gastrointestinal symptoms, body image, separate concerns for persons with or without a stoma, and sexual function (separate scale for men and women). Items for both questionnaires are evaluated using a 1week time frame (i.e., "during the past week") on a 4point scale ("not at all," "a little," "quite a bit," or "very much"), except for the global health scale of the QLQ30 , which is measured on a 7-point scale ranging from "very poor" to "excellent." For both the QLQ-C30 and QLQ-CR29, a higher score on the functional scales indicates better functioning, whereas a higher score on the symptom scales indicates worse symptoms.

Overall symptom burden will be assessed by the M.D. Anderson Symptom Inventory (MDASI) [37]. We selected the MDASI because it is brief and easy to use, and captures the most frequently reported disease- and treatment-related symptoms. The MDASI scale consists of 13 core symptom items (pain, fatigue, nausea, disturbed sleep, distress (emotional), shortness of breath, lack of appetite, drowsiness, dry mouth, sadness, vomiting, difficulty remembering, and numbness or tingling) and six interference items (general activity, mood, walking ability, normal work, relations with other people, and enjoyment of life). In addition to the 13 core symptoms, we will incorporate four additional symptoms that are specifically relevant in this clinical setting: mouth sores, hand-foot syndrome, diarrhea, and skin reaction at the site of irradiation.

Exercise motivation will be assessed using the TPB [25]. The key TPB constructs including attitudes, subjective norms, intention, and perceived behavioral control will be assessed using standardized items [38]. At the preNACRT time point, all patients will be asked to prospectively evaluate their motivation for the HIIT program. After NACRT, patients randomized to the exercise group will be asked to retrospectively evaluate their motivation for the HIIT program during NACRT and their prospective motivation for the exercise program post NACRT. Before surgery, patients in the exercise group will be asked to evaluate their retrospective motivation for the exercise program post NACRT, and all patients will be asked to evaluate their prospective motivation for exercising after surgery. All questions will be evaluated on a 5-point scale ranging from 1 ("not at all") to 5 ("very much").

\section{Feasibility and safety}

The feasibility and safety of the exercise intervention will be determined based on eligibility rate, recruitment rate, exercise adherence rate, assessment rate, and adverse events. The willingness of rectal cancer patients to be randomized to a supervised HIIT program during NACRT is unknown; however, based on the results of our feasibility study, we anticipate a recruitment rate $\geq 50 \%$ [16]. Moreover, we do not know the willingness of patients in the usual care group to return for all follow-up assessments; however, based on the results of our feasibility study, we anticipate a follow-up assessment rate $\geq 80 \%$ at each time point [16].

Exercise adherence during NACRT will be assessed by the number of exercise sessions attended out of 18 as well as adherence to the workload and duration of the highintensity intervals. Based on the results from our phase I study [16], we anticipate a median attendance rate to the supervised exercise training during NACRT $\geq 80 \%$. 
Exercise adherence to the unsupervised exercise training after NACRT will be assessed by self-report using the Godin Leisure-Time Exercise Questionnaire (GLTEQ) [39]. Based on our previous results [16] we anticipate that the mean number of moderate-to-vigorous-intensity exercise minutes post NACRT will be $\geq 222$ per week. Safety will be assessed by monitoring any serious adverse events that occur during exercise testing or the supervised exercise sessions. No serious exercise-related adverse events were observed in our previous study [16].

\section{Exploratory outcome measures}

Treatment toxicities will be assessed by clinical nurses on a weekly basis during NACRT using the National Cancer Institute Common Toxicity Criteria for Adverse Events Version 3.0. Treatment completion will be assessed as the number of patients completing $100 \%$ of their planned radiation dose within 1 week of the planned completion date using electronic medical records. Additionally, the number of patients receiving $\geq 80 \%$ of their planned chemotherapy dose will be recorded. Treatment response will be assessed by pathologic complete response as reported by the pathologist after reviewing the surgical sample. Surgical outcomes and complications will be obtained from medical records.

\section{Baseline descriptive variables}

Demographic variables will be collected from the baseline questionnaire and will include age, sex, marital status, education, income, employment status, and ethnicity. Behavioral variables will include smoking and physical activity. Physical activity (PA) will be assessed using the GLTEQ [39]. All participants will complete the GLTEQ at baseline (PA in the past month), post NACRT (unsupervised PA in the exercise training group; all PA in the usual care group), and pre surgery (all PA in both the exercise training and usual care groups). Medical variables will be abstracted from medical records at baseline and will include disease stage, chemotherapy protocol, and ostomy. Comorbidities and a list of medications will be collected in the baseline questionnaire.

Body composition/anthropometry will be assessed by height, weight, and waist and hip circumference [40, 41].

\section{Sample size}

Based on our feasibility study recruiting 18 patients in 6 months, we anticipate recruiting 60 patients over a 20month period and randomizing 30 patients to each group. Based on this sample size, our study has $80 \%$ power, with a two-tailed alpha $<0.05$, to detect a clinically meaningful effect of $3.5 \mathrm{ml} / \mathrm{kg} / \mathrm{min}$ on our primary outcome of $\mathrm{VO}_{2}$ peak post NACRT, assuming a standard deviation of $5.6 \mathrm{ml} / \mathrm{kg} / \mathrm{min}, 10 \%$ missing data, and adjustment for baseline value and other prognostic covariates [42]. This power may be sufficient for detecting differences in our secondary patient-reported outcomes if the effects are at least moderate (i.e., a standardized effect sizes of approximately $\geq d=0.60$ ). This power is unlikely sufficient for detecting potentially meaningful differences in any of the exploratory clinical outcomes. Given that the purpose of this phase II trial is to inform phase III trials, the patientreported and clinical outcomes will be interpreted for potential clinical significance based on the direction and magnitude of numerical differences.

\section{Data analysis}

We will use analysis of covariance (ANCOVA) at both the post-NACRT and pre-surgery time points to compare the two groups on all primary and secondary outcomes with adjustment for baseline value of the outcome as well as other potential covariates. All statistical analyses will be based on the intention-to-treat principle and include all patients with baseline and follow-up data. No missing data replacement strategies will be performed for this phase II trial as we anticipate $<10 \%$ missing data. Chi-square analyses will be used to explore between-group differences in the categorical and ordinal clinical outcomes. All analyses will be performed using SPSS (SPSS Inc., Chicago, IL, USA).

\section{Discussion}

NACRT is part of standard care for locally advanced rectal cancer and results in improvements in local recurrence rates and surgical outcomes. Furthermore, about $10-20 \%[43,44]$ of patients achieve a pathologic complete response to NACRT which is associated with better disease control and surgical outcomes [45]. Moreover, despite advances in supportive care management, NACRT still causes toxicities that can negatively impact outcomes for rectal cancer patients. Interventions to manage side effects, improve QoL and optimize treatment outcomes are needed. Exercise is a low-cost, low-toxicity intervention that improves symptom management, cardiorespiratory fitness, and QoL in several cancer patient groups; however, no definitive studies have examined the impact of exercise on outcomes for locally advanced rectal cancer patients. Loughney et al. [15] are currently conducting a randomized controlled trial in rectal cancer patients focused on the postNACRT phase and Moug et al. (ISRCTN62859294) are currently examining the effects of an unsupervised walking program during and after NACRT. We propose that an exercise training intervention both during and after NACRT may have additional benefits for symptom management, QoL, treatment outcomes, and possibly even survival (Fig 1).

The results from our phase I, single-arm study demonstrated that rectal cancer patients are willing and able to 
participate in a supervised, moderate-intensity, continuous exercise training intervention during NACRT followed by an unsupervised, moderate-intensity, continuous exercise training intervention after NACRT [16]. More specifically, we reported an excellent recruitment rate of 56\% (18/32 patients) over 6 months and a followup assessment rate of $>80 \%$ [16]. Moreover, the median attendance rate for the supervised exercise during NACRT was $83 \%$. After NACRT, patients completed an average of $222 \pm 155 \mathrm{~min} /$ week of unsupervised exercise [16]. No adverse events were observed and our evaluation was that even higher-intensity exercise would be feasible in this clinical setting [16]. Despite the exercise intervention, most health-related fitness outcomes and patient-reported outcomes declined during NACRT and recovered after NACRT [16]. Consequently, any benefit from exercise during NACRT is likely related to preventing declines in functioning. Moreover, patients reported that they experienced several benefits from exercise (e.g., physical fitness, QoL, self-esteem) but they also perceived some potential harms (e.g., worsening fatigue, diarrhea, skin irritation, hand-foot syndrome) [23]. We concluded that moderate-intensity, continuous exercise training during and after NACRT for rectal cancer is feasible and safe, and that phase II randomized controlled trials are needed to establish the benefits (and possible harms) of exercise in this clinical setting $[16,23]$.

The EXERT trial will be the first to evaluate the efficacy of exercise training for improving outcomes in rectal cancer patients during and after NACRT. Furthermore, the EXERT trial will establish the feasibility and safety of a HIIT program in this unique and challenging clinical setting. To date, most exercise oncology studies have focused on high-volume, continuous, moderate-to-vigorous-intensity exercise training [46]. HIIT is receiving attention in cancer patients [47-49] because of its ability to generate larger and more rapid improvements in maximal volume of oxygen consumption $\left(\mathrm{VO}_{2} \max \right)$ which may be a surrogate for important clinical outcomes such as QoL and survival $[9,26,28]$. Although moderate-intensity, continuous exercise training is beneficial for cancer patients, HIIT may be viewed as a potentially "high-risk, high-reward" exercise training intervention because its greater risk for safety and feasibility challenges may be offset by its greater potential for improved outcomes. Moreover, HIIT may be especially attractive in clinical settings, such as during NACRT or pre surgery, where shorter time frames are available for intervention delivery.

The EXERT trial will also be one of the few exercise oncology trials to examine the impact of exercise in the neoadjuvant setting and one of the few to include clinical cancer outcomes (e.g., treatment completion, pathologic complete response, post-surgical outcomes). In the rectal cancer setting, observational data suggest that cardiorespiratory fitness declines during NACRT and that pre-surgical cardiorespiratory fitness may predict post-surgical complications [29]. Initiating an exercise training intervention during NACRT could potentially optimize improvements in cardiorespiratory fitness which may result in fewer post-surgical complications and better post-surgical recovery when compared to an exercise training intervention initiated after NACRT. Additionally, poor compliance to external-beam radiation has been associated with an increased risk of disease recurrence and death [50]. If exercise is effective in managing symptoms and subsequently improving treatment compliance, radiation therapy may be optimized and result in better outcomes in this clinical setting. Finally, tumor hypoxia has been identified as a factor limiting the effectiveness of radiation therapy [51]. Preclinical models suggest that exercise may cause favorable changes in the vasculature of solid tumors thereby enhancing tumor oxygenation and possibly the effectiveness of radiation therapy [52-55]. Although these findings are intriguing, they have yet to be replicated in human clinical trials. Nevertheless, these are the outcomes that are most important to patients and clinicians and likely to drive changes in clinical practice.

To summarize, EXERT is the first phase II trial designed to generate preliminary efficacy data on the benefits and harms of exercise training, including clinical outcomes, in rectal cancer patients during and after NACRT. Additionally, EXERT will also establish the feasibility and safety of a supervised HIIT program in rectal cancer patients during NACRT. If the EXERT trial shows that exercise is safe, tolerable, and produces meaningful improvements in cardiorespiratory fitness, symptom management, QoL, and/or clinical outcomes, larger phase II and III trials designed to target these outcomes will be necessary to determine if exercise should be integrated in standard clinical care for this patient population.

\section{Trial status}

The trial opened for accrual in June of 2017 and is expected to be completed by June of 2019 .

\section{Additional file}

Additional file 1: SPIRIT 2013 Checklist: recommended items to address in a clinical trial protocol and related documents. (DOC $120 \mathrm{~kb}$ )

\section{Abbreviations}

EORTC: European Organisation of Research and Treatment of Cancer; GLTEQ: Godin Leisure-Time Exercise Questionnaire; HIIT: High-intensity interval training; MDASI: M.D. Anderson Symptom Inventory;

NACRT: Neoadjuvant chemoradiotherapy; PA: Physical activity; QoL: Quality of life; TPB: Theory of Planned Behavior; $\mathrm{VO}_{2}$ peak: Peak volume of oxygen consumption 


\section{Acknowledgements}

ARM is supported by a Frederick Banting and Charles Best Canada Graduate Scholarship from the Canadian Institutes of Health Research. KSC is supported by the Canada Research Chairs Program.

\section{Funding}

This work is supported by the Canadian Cancer Society (grant number 704680). The granting agency had no role in the design of the study and will not have a role in the collection, analysis, and interpretation of the data, nor in writing the manuscripts.

\section{Availability of data and materials}

Not applicable.

\section{Authors' contributions}

ARM and KSC made substantial contributions to the conception and design of the study and were involved in drafting the manuscript. NU and NGB made substantial contributions to the conception and design of the study and revised the manuscript for critically important intellectual content. DS, $K T, T N, K$, and $A F$ revised the manuscript for critically important intellectual content. All authors have read and approved the final manuscript.

\section{Ethics approval and consent to participate}

Ethics approval has been granted by the Health Research Ethics Board of Alberta (HREBA)-Cancer Committee (CC) (HREBA.CC-16-0986). All participants are required to provide written informed consent prior to participating in this study.

\section{Consent for publication}

Not applicable.

\section{Competing interests}

The authors declare that they have no competing interests.

\section{Publisher's Note}

Springer Nature remains neutral with regard to jurisdictional claims in published maps and institutional affiliations.

\section{Author details}

${ }^{1}$ Faculty of Kinesiology, Sport, and Recreation, University of Alberta, 1-113 University Hall, Van Vliet Complex, Edmonton, AB T6G 2H9, Canada. ${ }^{2}$ Department of Oncology, University of Alberta and Cross Cancer Institute, 11560 University Avenue, Edmonton, AB T6G 1Z2, Canada.

Received: 12 June 2017 Accepted: 7 December 2017

\section{Published online: 12 January 2018}

\section{References}

1. Patel SA, Ryan DP, Hong TS. Combined modality therapy for rectal cancer. Cancer J. 2016;22(3):211-17.

2. Sauer R, Becker H, Hohenberger W, Rodel C, Wittekind C, Fietkau R, et al. Preoperative versus postoperative chemoradiotherapy for rectal cancer. $\mathrm{N}$ Engl J Med. 2004;351(17):1731-40.

3. Sauer R, Liersch T, Merkel S, Fietkau R, Hohenberger W, Hess C, et al. Preoperative versus postoperative chemoradiotherapy for locally advanced rectal cancer: results of the German CAO/ARO/AIO-94 randomized phase III trial after a median follow-up of 11 years. J Clin Oncol. 2012;30(16):1926-33.

4. O'Gorman C, Denieffe S, Gooney M. Literature review: preoperative radiotherapy and rectal cancer-impact on acute symptom presentation and quality of life. J Clin Nurs. 2014;23(3-4):333-51.

5. Mishra SI, Scherer RW, Snyder C, Geigle PM, Berlanstein DR, Topaloglu O. Exercise interventions on health-related quality of life for people with cancer during active treatment. Cochrane Database of Syst Rev. 2012;8(8): CD008465.

6. Courneya KS, Segal RJ, Mackey JR, Gelmon K, Reid RD, Friedenreich CM, et al. Effects of aerobic and resistance exercise in breast cancer patients receiving adjuvant chemotherapy: a multicenter randomized controlled trial. J Clin Oncol. 2007;25(28):4396-404.

7. van Vulpen JK, Velthuis MJ, Steins Bisschop CN, Travier N, Van Den Buijs BJ, Backx FJ, et al. Effects of an exercise program in colon cancer patients undergoing chemotherapy. Med Sci Sports Exerc. 2016;48(5):767-75.
8. van Waart H, Stuiver MM, van Harten WH, Geleijn E, Kieffer JM, Buffart LM, et al. Effect of low-intensity physical activity and moderate- to high-intensity physical exercise during adjuvant chemotherapy on physical fitness, fatigue, and chemotherapy completion rates: results of the PACES randomized clinical trial. J Clin Oncol. 2015;33(17):1918-27.

9. Courneya KS, Sellar CM, Stevinson C, McNeely ML, Peddle CJ, Friedenreich $\mathrm{CM}$, et al. Randomized controlled trial of the effects of aerobic exercise on physical functioning and quality of life in lymphoma patients. J Clin Oncol. 2009;27(27):4605-12.

10. Courneya KS, Segal RJ, McKenzie DC, Dong H, Gelmon K, Friedenreich CM, et al. Effects of exercise during adjuvant chemotherapy on breast cancer outcomes. Med Sci Sports Exerc. 2014;46(9):1744-51.

11. Singh F, Newton RU, Galvao DA, Spry N, Baker MK. A systematic review of pre-surgical exercise intervention studies with cancer patients. Surg Oncol. 2013;22(2):92-104

12. Loughney L, West MA, Kemp GJ, Grocott MP, Jack S. Exercise intervention in people with cancer undergoing neoadjuvant cancer treatment and surgery: a systematic review. Eur J Surg Oncol. 2016;42(1):28-38.

13. Courneya KS. Physical activity and cancer survivorship: a simple framework for a complex field. Exerc Sport Sci Rev. 2014;42(3):102-09.

14. West MA, Loughney L, Lythgoe D, Barben CP, Sripadam R, Kemp GJ, et al. Effect of prehabilitation on objectively measured physical fitness after neoadjuvant treatment in preoperative rectal cancer patients: a blinded interventional pilot study. Br J Anaesth. 2015;114(2):244-51.

15. Loughney L, West MA, Kemp GJ, Rossiter HB, Burke SM, Cox T, et al. The effects of neoadjuvant chemoradiotherapy and an in-hospital exercise training programme on physical fitness and quality of life in locally advanced rectal cancer patients (The EMPOWER Trial): study protocol for a randomised controlled trial. Trials. 2016;17:24.

16. Morielli AR, Usmani N, Boule NG, Tankel K, Severin D, Nijjar T, et al. A phase I study examining the feasibility and safety of an aerobic exercise intervention in patients with rectal cancer during and after neoadjuvant chemoradiotherapy. Oncol Nurs Forum. 2016;43(3):352-62.

17. Heldens AF, Bongers BC, de Vos-Geelen J, van Meeteren NL, Lenssen AF, Feasibility and preliminary effectiveness of a physical exercise training program during neoadjuvant chemoradiotherapy in individual patients with rectal cancer prior to major elective surgery. Eur J Surg Oncol. 2016;42(9):1322-30.

18. Weston M, Weston KL, Prentis JM, Snowden CP. High-intensity interval training (HIT) for effective and time-efficient pre-surgical exercise interventions. Perioper Med. 2016;5:2.

19. Weston KS, Wisloff U, Coombes JS. High-intensity interval training in patients with lifestyle-induced cardiometabolic disease: a systematic review and meta-analysis. Br J Sports Med. 2014;48(16):1227-34.

20. Liubaoerijijin Y, Terada T, Fletcher K, Boule NG. Effect of aerobic exercise intensity on glycemic control in type 2 diabetes: a meta-analysis of head-tohead randomized trials. Acta Diabetol. 2016;53(5):769-81.

21. Adamsen L, Quist M, Andersen C, Moller T, Herrstedt J, Kronborg D, et al. Effect of a multimodal high intensity exercise intervention in cancer patients undergoing chemotherapy: randomised controlled trial. Br J Med. 2009;339:b3410.

22. Cardozo GG, Oliveira RB, Farinatti PT. Effects of high intensity interval versus moderate continuous training on markers of ventilatory and cardiac efficiency in coronary heart disease patients. Sci World J. 2015;2015:192479.

23. Morielli AR, Usmani N, Boule NG, Severin D, Tankel K, Nijjar T, et al. Exercise motivation in rectal cancer patients during and after neoadjuvant chemoradiotherapy. Support Care Cancer. 2016;24(7):2919-26.

24. Pescatello $L$, Arena R, Riebe $D$, Thompson WR. ACSM's guidelines for exercise testing and prescription, 9th ed. Philadelphia: Lippincott Williams \& Wilkins; 2014.

25. Ajzen I. The theory of planned behavior. Organ Behav Hum Decis Process. 1991;50:179-211.

26. Courneya KS, Mackey JR, Bell GJ, Jones LW, Field CJ, Fairey AS. Randomized controlled trial of exercise training in postmenopausal breast cancer survivors: cardiopulmonary and quality of life outcomes. J Clin Oncol. 2003; 21(9):1660-68.

27. Kodama S, Saito K, Tanaka S, Maki M, Yachi Y, Asumi M, et al. Cardiorespiratory fitness as a quantitative predictor of all-cause mortality and cardiovascular events in healthy men and women: a meta-analysis. J Am Med Assoc. 2009;301(19):2024-35.

28. Schmid D, Leitzmann M. Cardiorespiratory fitness as predictor of cancer mortality: a systematic review and meta-analysis. Ann Oncol. 2015;26(2):272-78. 
29. West MA, Parry MG, Lythgoe D, Barben CP, Kemp GJ, Grocott MP, Jack S. Cardiopulmonary exercise testing for the prediction of morbidity risk after rectal cancer surgery. Br J Surg. 2014;101(9):1166-72.

30. McInnis K, Balady GJ. Comparison of submaximal exercise responses using the Bruce vs modified Bruce protocols. Med Sci Sports Exerci. 1994;26(1): 103-7.

31. Thompson WR, Gordon NF, Pescatello LS. ACSM's guidelines for exercise testing and prescription, 8th ed. Philadelphia: Lippincott Williams \& Wilkins; 2010

32. Jones CJ, Rikli RE. Measuring functional fitness of older adults. J Active Aging. 2002;1:24-30.

33. Rikli RE, Jones CJ. Development and validation of a functional fitness test for community-residing older adults. J Aging Phys Act. 1999;7:129-61.

34. Aaronson NK, Ahmedzai S, Bergman B, Bullinger M, Cull A, Duez NJ, et al. The European Organization for Research and Treatment of Cancer QLQ-C30: a quality-of-life instrument for use in international clinical trials in oncology. J Natl Cancer Inst. 1993;85(5):365-76.

35. Giesinger JM, Kieffer JM, Fayers PM, Groenvold M, Petersen MA, Scott NW, et al. Replication and validation of higher order models demonstrated that a summary score for the EORTC QLQ-C30 is robust. J Clin Epidemiol. 2016;69:79-88.

36. Whistance RN, Conroy T, Chie W, Costantini A, Sezer O, Koller M, et al. Clinical and psychometric validation of the EORTC QLQ-CR29 questionnaire module to assess health-related quality of life in patients with colorectal cancer. Eur J Cancer. 2009:45(17):3017-26.

37. Cleeland CS, Mendoza TR, Wang XS, Chou C, Harle MT, Morrissey M, Engstrom MC. Assessing symptom distress in cancer patients. Cancer. 2000; 89(7):1634-46

38. Constructing a Theory of Planned Behavior questionnaire: conceptual and methodological considerations. 2006. https://people.umass.edu/aizen/pdf/ tpb.measurement.pdf. Accessed 8 June 2017.

39. Godin G, Shephard RJ. A simple method to assess exercise behavior in the community. Can J Appl Sport Sci. 1985;10(3):141-46.

40. World Health Organization. Waist circumference and waist-hip ratio: report of a WHO expert consultation, Geneva, 8-11 December 2008. Geneva: World Health Organization; 2011. p. 39.

41. National Health Institute. The practical guide identification, evaluation, and treatment of overweight and obesity in adults. NIH Publication Number DO-4084 2000:1-80

42. Borm GF, Fransen J, Lemmens WA. A simple sample size formula for analysis of covariance in randomized clinical trials. J Clin Epidemiol. 2007; 60(12):1234-38.

43. Habr-Gama A, de Souza PM, Ribeiro Jr U, Nadalin W, Gansl R, Sousa AH, et al. Low rectal cancer: impact of radiation and chemotherapy on surgical treatment. Dis Colon Rectum. 1998;41(9):1087-96.

44. Kuo LJ, Liu MC, Jian JJ, Horng CF, Cheng TI, Chen CM, et al. Is final TNM staging a predictor for survival in locally advanced rectal cancer after preoperative chemoradiation therapy? Ann Surg Oncol. 2007;14(10):2766-72.

45. Habr-Gama A, Perez R, Nadalin W, Nahas SC, Ribeiro U, Silva e Sousa AH, et al. Long-term results of preoperative chemoradiation for distal rectal cancer correlation between final stage and survival. J Gastrointest Surg. 2005;9(1): 90-101.

46. Jones LW, Liang Y, Pituskin EN, Battaglini CL, Scott JM, Hornsby WE, Haykowsky M. Effect of exercise training on peak oxygen consumption in patients with cancer: a meta-analysis. Oncologist. 2011;16(1):112-20.

47. Licker M, Karenovics W, Diaper J, Fresard I, Triponez F, Ellenberger C, et al. Shortterm preoperative high-intensity interval training in patients awaiting lung cancer surgery: a randomized controlled trial. J Thorac Oncol. 2017;12(2):323-33.

48. Adams SC, DeLorey DS, Davenport MH, Stickland MK, Fairey AS, North S, et al. Effects of high-intensity aerobic interval training on cardiovascular disease risk in testicular cancer survivors: a phase 2 randomized controlled trial. Cancer. 2017;123(20):4057-65.

49. Devin JL, Sax AT, Hughes Gl, Jenkins DG, Aitken JF, Chambers SK, et al. The influence of high-intensity compared with moderate-intensity exercise training on cardiorespiratory fitness and body composition in colorectal cancer survivors: a randomised controlled trial. J Cancer Surviv. 2016;10(3):467-79.

50. Ohri N, Rapkin BD, Guha C, Kalnicki S, Garg M. Radiotherapy noncompliance and clinical outcomes in an urban academic cancer center. Int J Radiat Oncol Biol Phys. 2016;95(2):563-70.

51. Jordan BF, Sonveaux P. Targeting tumor perfusion and oxygenation to improve the outcome of anticancer therapy. Front Pharmacol. 2012;3:94.

52. Betof AS, Lascola CD, Weitzel D, Landon C, Scarbrough PM, Devi GR, Palmer $G$, et al. Modulation of murine breast tumor vascularity, hypoxia and chemotherapeutic response by exercise. J Natl Cancer Inst. 2015; 107(5):dvj040.

53. McCullough DJ, Stabley JN, Siemann DW, Behnke BJ. Modulation of blood flow, hypoxia, and vascular function in orthotopic prostate tumors during exercise. J Natl Cancer Inst. 2014;106(4):dju036.

54. McCullough DJ, Nguyen LMD, Siemann DW, Behnke BJ. Effects of exercise training on tumor hypoxia and vascular function in the rodent preclinical orthotopic prostate cancer model. J Appl Physiol. 2013;115(5):1846-54.

55. Jones LW, Antonelli J, Masko EM, Broadwater G, Lascola CD, Fels D, et al. Exercise modulation of the host-tumor interaction in an orthotopic model of murine prostate cancer. J Appl Physiol. 2012;113(2):263-72.

\section{Submit your next manuscript to BioMed Central and we will help you at every step:}

- We accept pre-submission inquiries

- Our selector tool helps you to find the most relevant journal

- We provide round the clock customer support

- Convenient online submission

- Thorough peer review

- Inclusion in PubMed and all major indexing services

- Maximum visibility for your research

Submit your manuscript at www.biomedcentral.com/submit
Biomed Central 